\title{
La formación del profesorado basada en redes de aprendizaje virtual: aplicación de la técnica dafo.
}

\author{
Pilar Colás Bravo Juan de Pablos Pons \\ pcolascus.es jpabloseus.es \\ Equipo de Investigación, Evaluación y \\ Tecnología Educativa \\ Universidad de Sevilla
}

\section{Introducción.}

Esta aportación está relacionada con el desarrollo de un proyecto de investigación I+D [1] que pretende promover y potenciar la integración de recursos digitales en red en contextos de aula y en los centros educativos, a través de desarrollos curriculares específicos en las Enseñanzas Secundarias y Bachillerato.

La formación del profesorado en las tecnologías de la información y la comunicación (TICs) es una de las áreas prioritarias en la actual sociedad del conocimiento, sin duda un aspecto imprescindible para lograr la plena alfabetización digital de la ciudadanía. Pero también es clave para lograr la plena incorporación de las TICS a la enseñanza y el aprendizaje. Anteriores investigaciones, realizadas por el equipo de investigación de los autores, sobre los procesos de implantación de las nuevas tecnologías en los centros escolares de primaria y secundaria señalan, como motivos de la escasa integración de las TICs en los centros escolares, la insuficiente formación del profesorado en TICs y la falta de referencias para desarrollar aplicaciones educativas con recursos tecnológicos digitales (De Pablos 2000 y Colás, 2001/2002).

Nuestra aportación se propone explorar diferentes modelos y formatos de formación del profesorado haciendo uso de las posibilidades que ofrecen los propios recursos tecnológicos. Se concreta en una propuesta de formación virtual destinada a capacitar al profesorado para integrar las TICs dentro de sus competencias y actividades docentes desde una perspectiva colaborativa y contextualizada, en un perfil específico como es la Educación Física en la Enseñanza Secundaria Obligatoria y el

Bachillerato. Para su desarrollo se hace uso de la técnica DAFO (Debilidades, Amenazas, Fortalezas y Oportunidades), ya que se trata de una herramienta de análisis que permite profundizar en los entornos o contextos en los que se produce una intervención o aplicación.

\section{La formación del profesorado y las TICs.}

La formación del profesorado a la largo del tiempo ha ido evolucionando y sustentándose en distintas concepciones, y modelos, acordes con los paradigmas científicos dominantes de cada momento. En la actualidad parece prevalecer el modelo de formación del profesorado desarrollado en centros. Buscando así, que el profesorado contextualice y sitúe las propuestas de formación y mejora que se proponen.

Por otra parte, la fuerte presencia de las TICs en las sociedades avanzadas, la incorporación de una visión cultural de la educación y las aplicaciones de teorías psicológicas basadas preferentemente en perspectivas socioculturales (Daniels, 2003) permiten la opción de plantear la formación del profesorado desde nuevos ángulos y con nuevos enfoques. La teoría social que se ha desarrollado a partir de las aportaciones de Vygotsky constituye un marco de referencia muy rico para interpretar y 
desarrollar los procesos de transformación social, como es el caso de la educación. Una manera de comprender en profundidad las aportaciones de Vygotsky es verlo como un psicólogo cultural. Michael Cole (1999) al hacer un planteamiento dirigido a repensar el papel de la psicología propone emprender la marcha por el camino no transitado: el que sitúa a la cultura en el mismo nivel de la biología y la sociedad como factores decisivos en el desarrollo de la naturaleza humana individual. Con este referente es posible subrayar conceptos que aportan nuevas vertientes como el papel de las "acciones mediadas" en contextos educativos concretos; el carácter de los agentes de aprendizaje como sujetos activos de su propio desarrollo, aunque en entornos no totalmente controlados; la naturaleza emergente de la mente, o las características de la metodología genética, que permite integrar estos conceptos en estudios de campo.

A partir de la utilización de conceptos como mediación, metodología genética o construcción social y activa del conocimiento, el análisis de las tecnologías y su uso en los contextos educativos cobra nuevas perspectivas. Asimismo, las TICs permiten nuevas posibilidades y nuevos formatos educativos, ya que rompen las barreras limitadoras de las disciplinas curriculares al permitir aprender de forma interdisciplinar y abierta. También posibilitan el hecho de "aprender en la multiculturalidad" y amplían y multiplican los referentes formativos. Estos nuevos contextos formativos exigen cambios en las competencias y roles del profesor (De Pablos, 2003). El profesor ya no es la fuente única del saber ya que "comparte" estas competencias con textos, especialistas, expertos, compañeros, personas de otras culturas, documentales, etc.

J.M. Touriñan (2001: 222) apunta que el papel fundamental del profesor en estos nuevos entornos es el de actuar como guía e instrumento del aprendizaje significativo a través de la red. Es decir, una labor centrada en ayudar a construir conocimiento en Red. El profesor se convierte en "un gestor de la formación". Por lo tanto, el que se ocupa de gestionar las capacidades, habilidades y conocimientos de los aprendices, detectando, motivando y aprovechando tanto individualmente como colectivamente sus posibilidades de aprendizaje (Colás, 2003b: 33).

Las potencialidades educativas de redes como Internet, obligan a replantear muy seriamente la dimensión individual y colectiva de los procesos de enseñanza-aprendizaje, los ritmos o tiempos de aprendizaje, las nuevas formas de estructurar la información para la construcción de conocimiento, las tareas y competencias docentes, etc. La problemática pedagógica de las TICs es tratada actualmente por numerosos autores (Hannafin y Kim, 2003; Reigeluth,2003; Lynch y Lang, 2004; Watson, 2001; Jedeskog y Nissen, 2004). Pero no podemos olvidar que la tecnología en sí misma no supone una oferta pedagógica como tal, sino que su validez educativa estriba en el uso que los agentes educativos o las comunidades educativas hagan de ella. De ahí que la formación de profesorado en TICs y con TICs se convierta en uno de los factores claves para su uso y utilización en los sistemas de formación tanto reglada como no reglada. Ello implica la construcción de una nueva pedagogía tecnológica que posibilite e integre lo local con lo global y que haga compatible la formación en centros con la constitución de redes temáticas especializadas que construyan y reconstruyan conocimientos y saberes disciplinares. Este potencial ha de canalizarse a través de la creación de nuevos modelos y de formas de gestión pedagógica que permitan la explotación de las posibilidades interactivas del espacio virtual.

\section{El aprendizaje en Red. Líneas de investigación}

Los términos Internet y aprendizaje configuran un ámbito científico de interesantes aplicaciones educativas en el momento actual. Internet está generando una amplia variedad de estudios, desde una perspectiva educativa, observándose un progresivo aumento de la producción científica en estos terrenos [2] (Hannafin, y otros 2001; Oliver y otros, 2001, Weert y Pilot. 2003).

Las aportaciones científicas de carácter empírico (preferentemente en el ámbito anglosajón) prestan especial atención a cuatro aspectos (Colás, 2002, 2003b): 1) Internet y aprendizaje, 2) Aprendizaje e Interacción comunicativa a través de la red, 3) Gestión del aprendizaje en la red,4) Impacto de la red en aprendizajes socio-culturales. 
1) Internet y aprendizaje. Esta línea de trabajo estudia la influencia de Internet en el aprendizaje desde el punto de vista de: a) nuevo entorno que produce cambios en relación con los sistemas clásicos de enseñanza, b) procesos cognitivos que se ponen en uso mediante el manejo de Internet, c) contextos educativos en los que la Web se manifiesta útil y d) aprendizaje de los alumnos en contextos de referencia que ofrece la Web. Los trabajos de Hannafin y Kim, (2003) Booth y Hultén (2003), Hill (1999), Nahl (1998), Locatis y Weisberg (1997) son muestras de aportaciones a este campo.

2) Aprendizaje e Interacción comunicativa a través de la Red. Las potencialidades para el aprendizaje que proporciona la dimensión interactiva de Internet es el núcleo central de estas aportaciones. Se investigan las distintas opciones que ofrece Internet, desde el punto de vista de la participación, en la generación de conocimiento. Estos estudios exploran las interacciones comunicativas en redes de aprendizaje. Se perfilan cuatro áreas de trabajo: a) Actividades en Red organizadas en torno a un ciclo de aprendizaje. b) Diseño, planificación y puesta en marcha de cursos de formación a distancia, apoyados en plataformas digitales. c) Realización de estudios o investigaciones de forma colaborativa a través de la Red. d) Colaboración y participación para crear contextos propios de aprendizaje. Los trabajos de Booth y Hulten, (2003), Gillian,(2003), Dillenbourg, (1999), y Hooper (1992) se sitúan bajo este enfoque.

3) Gestión del Aprendizaje en la Red. Esta línea estudia los procesos metodológicos y secuencias operativas que se llevan a cabo en los procesos formativos cuando se utiliza Internet como fuente de información. Tres aspectos son objeto de investigación: a) Generación de cuestiones, es decir, cómo los profesores plantean las tareas a resolver. b) Búsquedas de contenidos específicos, como los estudiantes traducen y concretan las demandas del profesor en cuestiones o temáticas concretas para su localización en Internet. c) Acceso a la información, es decir, como los alumnos estructuran los recursos y tiempo a través de la Web. Steeples y Jones (2002), McConnell, (2000), Harasim y otros. (1997), Freeman y Liu, (1996) y Rysavy y Sales,( 1991), entre otros, trabajan en esta línea de investigación.

4) Impacto de la Red en Aprendizajes Socio-Culturales. Bajo esta perspectiva se desarrollan las siguientes temáticas: a) Impacto de las TICs (Tecnologías de la Información y la Comunicación) en la forma que los estudiantes perciben a los otros, en lo que ellos creen sobre otras culturas, y cómo los estudiantes revisan su propia visión del mundo. b) Cambios que propicia Internet en valores, actitudes y creencias. c) Cambios en las formas de expresión. d) Procesos de aprendizaje desde la óptica de la construcción social del aprendizaje a través del conocimiento virtual. e) Cómo se constituyen grupos sociales de forma virtual como resultado de la telecomunicación y qué motivaciones conforman estos grupos. Trabajos como los de De Pablos, (1999, 2001), Jonassen, y Rohrer-Murphy (1999), Kuutti, (1996), Jonassen y Rohrer-Murphy ( 1999) dan fe de los desarrollos de esta línea de investigación.

Todas estas aportaciones son iniciativas de comunidades científico- educativas dirigidas a estudiar el impacto de las TICs en los procesos educativos y formativos. En estas líneas de trabajo subyace la idea de que las tecnologías aumentan el rendimiento del alumno a través de la mejora de la calidad de los procesos instructivos. De ahí que predominen estudios sobre la efectividad de las tecnologías en el aprendizaje (Morales y otros, 2000).

Los últimos desarrollos, sin embargo, se orientan a la implantación e integración de las tecnologías en los centros y las clases. Ello obedece a la creencia y asunción de que los beneficios de las tecnologías en los centros escolares y en las aulas sólo son posibles a través de una idónea contextualización de las mismas. Se traslada el centro de atención hacia los procesos de integración de las TICs en los sistemas y organizaciones educativas. Se abre por tanto una nueva línea de trabajo en el terreno educativo, centrada en la incorporación de las TICs en los sistemas educativos, centros escolares y aulas, a través de los correspondientes diseños curriculares.

Es precisamente en esta perspectiva en la que se inserta el proyecto I+D que venimos desarrollando (Colás, 2003) en el que se combinan objetivos formativos de distintas líneas científicas: creación de contextos formativos para el desarrollo profesional docente, diseño y puesta en marcha de cursos 
teleformativos haciendo uso de las potencialidades de la red, como por ejemplo la colaboración y participación para crear contextos propios de aprendizaje que posibiliten la realización de estudios o investigaciones de forma colaborativa a través de la Red o la constitución de grupos sociales de forma virtual como resultado de intereses y motivaciones compartidos.

\section{Modelos de formación del profesorado con TICs: La formación basada en redes de aprendizaje virtual}

Es frecuente observar como los modelos de formación del profesorado a través de plataformas digitales o teleformación adoptan fórmulas y técnicas clásicas que se repiten en un mayor o menor grado. En ellas se plantea el aprendizaje y la formación como una reproducción de los conocimientos que los expertos lanzan y plantean con mayor o menor grado de acierto y planificación didáctica. Son escasos los modelos alternativos de formación que representen una concepción más abierta e interpretativa de la formación docente. Es decir, el profesor además de los contenidos debe recibir las herramientas para observar, interpretar, reflexionar y tomar decisiones haciéndole más protagonista y responsable de sus acciones y de sus aprendizajes. Pero tampoco están habitualmente presentes otros modelos que asuman que el profesor es un agente de transformación cultural, o un agente que promueve la interacción hacia una cultura global-plural. La perspectiva o enfoque sociocultural aplicado a la formación del profesorado fundamenta teóricamente estas dos últimas perspectivas, más acordes, por otra parte, con las posibilidades y el sentido de las TICs en el mundo actual.

En nuestra propuesta se plantea un modelo de formación del profesorado fundamentado en el enfoque sociocultural para promover y potenciar la utilización de recursos digitales en red, tanto en las aulas como en los centros educativos a través de desarrollos curriculares específicos del campo de la Educación Física, en la Enseñanza Secundaria Obligatoria (ESO) y Bachillerato. En este modelo el profesorado tiene un peso y un papel protagonista en el análisis, interpretación y decisiones sobre su realidad educativa. El profesor es considerado como el principal agente de un proceso de transformación que alude a la cultura del centro, y ello mediante un uso de las TICs que potencien la interculturalidad, la interacción y la conexión con un mundo global. Para ello se ha elaborado un modelo de formación que incluye cuatro fases de desarrollo consecutivas y que inciden en distintos tipos de aprendizaje:

a) APRENDIZAJE INSTRUMENTAL. Implica el manejo de una plataforma digital (virtual profe4) y navegación por e-recursos educativos. Esta fase es punto de partida para poder hacer uso de la plataforma para su formación y, a la vez, representa un ámbito de experimentación como recurso de aprendizaje.

b) APRENDIZAJE COLABORATIVO. El trabajo en red posibilita la creación de una red temática de profesores, en nuestro caso pertenecientes al perfil de Educación Física, que asuma el desarrollo de aplicaciones en los centros educativos y en el aula. Esta red se construye en base al diagnóstico de la situación de su centro en relación a las TICs, con el propósito de construir una propuesta y plan de innovación. En esta forma de aprendizaje se hace realidad la idea de que el profesor construya su aprendizaje e intervenga en la realidad a partir de su reflexión y acción. La metodología, basada en el diseño y desarrollo de proyectos, pretende motivar, estimulando la autonomía, iniciativa, creatividad para la aplicación del conocimiento.

c) CREACIÓN E INNOVACIÓN EN SUS PRÁCTICAS. La incorporación de las TICs a los procesos de enseñanza-aprendizaje desde un enfoque sociocultural llevará a la incorporación de nuevos conceptos mediadores y facilitadores de interpretaciones alternativas de la realidad y, consecuentemente, propiciará aplicaciones innovadoras y potentes de estas nuevas herramientas y recursos en la educación.

d) TRANSFORMACIÓN DISCIPLINAR. En un cuarto momento las TICs se convierten en un medio que llevará a la necesidad de repensar la formación tradicional de una determinada disciplina. Se trata de reflexionar y encontrar nuevas conceptos y formas de entendimiento de la formación. Se trata en 
última instancia de repensar el concepto formativo subyacente en la propia disciplina y el papel que ésta tiene en la formación de ciudadanos.

La formación docente basada en redes de aprendizaje virtual se asume y se desarrolla en base a dos ejes: "La construcción social del aprendizaje en la red" y "la formación del profesorado en centros mediante la creación de redes de aprendizaje". Este modelo de formación parte de la creación de comunidades docentes de aprendizaje colaborativo como base para la formación permanente del profesorado. Las redes de aprendizaje y formación virtual aportan marcos en los que los profesores a través de su participación, implicación y colaboración con otros profesores construyen colectivamente conocimiento sobre currículo y TICs. Este formato se apoya en la idea de que el aprendizaje es un proceso intrínsecamente social, basado en la interacción y cooperación entre personas y que pasa, en buena parte, por la participación junto con otros en situaciones reales en las que se pone en juego y se utiliza de forma funcional y auténtica el conocimiento. Los procesos de aprendizaje y formación pueden adoptar formas diversas, como la elaboración de proyectos, el análisis de casos, la resolución de situaciones problema o la presentación de productos que serán expuestos públicamente.

Más allá de formas concretas que puedan adoptar, lo esencial es que estos procesos remitan a situaciones, actividades y tareas auténticas y relevantes para los participantes. Auténticas en el sentido de que constituyan situaciones y problemáticas reales y relevantes, en tanto que se remiten a problemas o temáticas valoradas culturalmente desde la perspectiva docente.

Por otra parte las TICs posibilitan la creación de redes para distintos propósitos formativos. En nuestro caso se plantean para el desarrollo y uso de las tecnologías en los centros educativos, para la gestión del cambio, compartir el conocimiento, y construir conocimientos de forma colaborativa. La implicación y la participación activa de los profesores potencia la motivación, la comunicación y la construcción de una nueva cultura de formación permanente en red. A la vez que propicia una capacitación para el cambio y la autogestión del mismo, basándose en la cooperación interna y externa.

Se trata en suma de experimentar modelos formativos con TICs que provoquen transformaciones e innovaciones educativas extensivas a colectivos docentes cada vez más amplios, y que éstas se orienten para hacer de los profesores agentes activos y transformadores de sus prácticas. Con este propósito planteamos una adaptación de la técnica DAFO como herramienta para la creación y cooperación de redes de aprendizaje. Las redes de aprendizaje y formación permanente del profesorado se plantean como acciones formativas orientadas al desarrollo y uso de las tecnologías en los centros educativos.

\section{La técnica DAFO aplicada a la formación docente on line.}

La técnica DAFO responde a las siglas de Debilidades, Amenazas, Fortalezas y Oportunidades. Es una técnica que se aplica en los estudios prospectivos sobre dinámicas sociales participativas. Desde 1994 se está adoptando en Europa por grupos cada vez más numerosos con aplicaciones en muy diversos ámbitos sociales y económicos. Esta técnica alcanza en los últimos años una gran relevancia en la planificación estratégica y en el diagnóstico de necesidades, sin olvidar las propuestas de intervención de forma consensuada y participativa. Esta metodología es útil cuando se pretende impulsar transformaciones estructurales y dinamizar el cambio, elaborar proyectos de acción, así como crear redes y tramas de colaboración.

La Técnica DAFO metodológicamente se desarrolla en base a cuestiones que se plantean a grupos de expertos con el propósito de que diagnostiquen la situación presente, proyecten situaciones futuras y prevean acciones posibles considerando los condicionantes tanto en positivo como en negativo que rodea la temática a abordar. Tradicionalmente se concreta en preguntas que corresponden a criterios internos (Fortalezas y Debilidades) y externos (Oportunidades y Amenazas).

La incorporación de la técnica DAFO a la formación docente virtual pretende que los profesores 
reflexionen y valoren las problemáticas específicas de sus centros, contextos y culturas escolares en la integración de las TICs a sus tareas docentes. Que analicen el potencial de sus contextos y situaciones en la integración de las TICs, así como los principales "handicaps" derivados de la propia tradición escolar. Y tras este diagnóstico propongan actuaciones innovadoras en su aula, haciendo uso de los recursos educativos disponibles en Internet. Esta metodología de trabajo se lleva a cabo a través de foros virtuales. En la experiencia que estamos desarrollando en el marco del proyecto de investigación antes señalado, participan en torno a 60 profesores de educación secundaria y bachillerato, especialistas en Educación Física.

Mediante esta técnica se pretenden tres objetivos básicos de aprendizaje:

1) Realizar un diagnóstico de la situación de su centro en relación con las TICS.

2) Crear una red temática de profesores de Educación Física para el desarrollo de aplicaciones de TICS en los Centros Educativos.

3) Elaborar una propuesta de innovación y desarrollo de las TICS en los centros educativos.

El proceso metodológico se desarrolla en base al Foro de discusión "Problemática de las TICs en los Centros Educativos". A él tienen que aportar tres debilidades que observen en sus respectivos centros que dificulten la integración de las TICs en el currículo, tres fortalezas, tres amenazas y una propuesta de acción.

A partir de la recogida de datos vinculada a esta problemática y las temáticas de acción generadas, se les insta a que creen sus redes de acción e intervención de forma colaborativa en función de coincidencias en propuestas o puntos de vista. Para el trabajo de los grupos se generan foros temáticos que sirven para debatir, madurar, y configurar un proyecto de innovación a desarrollar de forma colaborativa en red. Los foros específicos creados sobre proyectos concretos planteados, sirven, por tanto, para debatir, definir y concretar las propuestas iniciales.

La aplicación de esta técnica DAFO a la formación virtual de los docentes tiene aspectos positivos que deben ser resaltados:

a) Posibilitar y propiciar la participación de docentes que pueden ser representativos de diferentes perfiles, en función del centro en el que trabajan y del conocimiento y experiencia que poseen.

b) Aportar una visión global de la realidad educativa, así como identificar aspectos relevantes a considerar en los cambios o propuestas de innovación.

c) Al realizarse sobre contextos grupales favorece la participación y el debate de los docentes implicados, recogiendo todas las opiniones.

d) Facilitar la elaboración de proyectos de innovación en las prácticas educativas de forma colaborativa, al articular las iniciativas de acción propuestas por los docentes. La elaboración de proyectos aparece como consecuencia lógica del análisis de necesidades iniciales, elaborado mediante la técnica DAFO.

e) Propiciar una construcción social del aprendizaje, de planes de acción para la mejora, basadas en el análisis concreto y preciso de la realidad escolar.

f) Constituye una herramienta útil en las acciones formativas, especialmente orientadas al desarrollo y uso de tecnologías en los centros educativos y en las prácticas docentes.

\section{A modo de conclusión}

Esta experiencia de formación del profesorado de secundaria en TICs apoyada en la técnica DAFO, 
nos permite explorar y experimentar nuevos conceptos de formación basados en planteamientos teóricos alternativos. La apertura de nuevos ángulos para observar los problemas desde nuevas perspectivas. Esta experiencia incide en la capacitación del profesorado, en base a la constitución de comunidades de aprendizaje y la organización de redes de profesores comprometidos con la innovación, tratando de optimizar las posibilidades que brindan las TICs.

Una cuestión de gran trascendencia, que está a la base del desarrollo de proyectos como el que aquí se presenta, consiste en atraer al profesorado hacia propuestas dirigidas a la creación de una nueva cultura de organización del trabajo. Más allá de la utilización de espacios y tiempo de manera convencional. Y ello, como resultado de un aprendizaje y formación previa básica en el uso de plataformas digitales y la configuración de redes docentes que se gestan a través de procesos de cooperación interna y externa.

\section{Bibliografía.}

BOOTH, S. \& HULTEN, M. (2003). Opening dimensions of variation: An empirical study of learning in a web-based discussion. Instructional Science 31(1-2), 65-86

COLÁS, P. (Dir.)(2003). Utilización de las TICS en el desarrollo Curricular de la Educación Física: Diseño, Implementación y Evaluación de un modelo de formación para el Profesorado de Secundaria y Bachillerato. Proyecto I+D (Referencia : BSO2003-00340/PSCE) subvencionado por el Ministerio de Ciencia y Tecnología en convocatoria pública (BOE 31/10/2002)

COLÁS, P. (2003b). Internet y Aprendizaje en la sociedad del conocimiento. Comunicar, 20, 31-35.

COLÁS, P. (2002). La investigación educativa en la (nueva) cultura de la sociedad del conocimiento. XXI. Revista de Educación. 4, 77- 95.

COLÁS, P. (2001/2002). Evaluación de la implantación de tecnologías de la información y la comunicación en centros escolares. Qurrículum, 15, 91-117.

COLE, M. (1999). Psicología cultural. Madrid, Ed. Morata.

DE PABLOS, J. (1999). Las nuevas tecnologías y la construcción de la identidad cultural. El cambio educativo para el siglo XXI. Bordón, 5(4), 417-433.

DE PABLOS, J. (2000). Los centros de profesorado y su incidencia en la implantación de las nuevas tecnologías en el sistema educativo andaluz. En Lorenzo y otros (Coord). Las organizaciones educativas en la sociedad neoliberal. Granada: Grupo Editorial Universitario.

DE PABLOS, J. (2001). Los estudios culturales y la comunicación. Algunas herramientas conceptuales para interpretar la mediación tecnológica. En Area. M. (Coord). Educar en la Sociedad de la Información. Bilbao: Descleé de Brouwer.

DE PABLOS, J. (2003). La tecnología educativa hoy no es como ayer: nuevos enfoques, nuevas miradas. Tecnología y Comunicación Educativa (Instituto Latinoamericano de la Comunicación Educativa), número 37, (I)

DANIELS, H. (2003). Vygotky y la pedagogía. Barcelona, Paidós.

DILLENBOURG, P. (ed.) (1999). Collaborative Learning: Cognitive and ComputationalApproaches. London: Pergamon.

FREEMAN, K. Y LIU, M. (1996).The Importance of Computer Experience, Learning Processes, and Communication Patterns in Multivultural Networking. Educational Technology Research and Development 44 (1), 43-59. 
GILLIAN, R.(2003) Teaching using the Web: Conceptions and approaches from a phenomenographic perspective. Instructional Science 31: 127-150, 2003

HILL, J. ( 1999). A conceptual framework for Understanding Information Seeking in Open-Ended Information Systems. Educational Technology Research and Development 47 (1), 5-28.

HANNAFIN, M Y KIM, M. (2003). In search of a future: A critical analysis of research on webbased teaching and learning. Instructional Science,31(4-5), 347-351.

HANNAFIN, M. Y OTROS. (2001). Research on and research with emerging Technologies. Bloomington. AECT ( Association for Educational Communication and Technology).

HARASIM, L., HILTZ, S.R., TELES, L. \& TUROFF, M. (1997). Learning Networks. Cambridge,Massachusetts, The MIT Press.

HOOPER, S. (1992). Cooperative learning and computer-based instruction. Educational Technology Research and Development 40 (3), 21-38.

JONASSEN, D. Y ROHRER-MURPHY, L (1999). Activity Theory as a Framework for Designing Constructivist Learning Enviroments. . Educational Technology Research and Development 47 (1), 61-79.

JEDESKOG , G. Y NISSEN, J. (2004). ICT in the Classroom: Is Doing More Important than Knowing?. Education and Information Technologies, 9 (1): 37-45

KUUTTI, K. (1996). Activity theory as a potential framework for human-computer interaction research. En Nardi, B.A. ( Ed).Context and consciousness: Activity theory and human -computer interaction. Cambridge, M.A. MIT Press.

LYNCH, M Y LANG, M(2004) The Online Educator. A Guide to Creating the Virtual Classroom. Education and Information Technologies, 9 (1): 91-93.

LOCATIS, C. Y WEISBERG, M. (1997). Distributed learning and the Internet. Contemporary Education, 56 (2),100-130.

MCCONNELL, D. (2000). Implementing Computer Supported Cooperative Learning, 2nd ed.London: Kogan Page.

NAHL, D. (1998). Learning the Internet and the structure of information behaviour. Journal of the American Society for Information Science, 49 (11), 1017-1023.

OLIVER, K. Y HANNAFIN, M. (2001). Developing and Refining Mental Models in Open-Ended Learning Environments: A case study. Educational Technology Research and Development 49 (4): 5 32 .

REIGELUTH,CH.(2003). Knowledge building for use of the internet in education. Instructional Science 31, (4-5): 341-346.

RYSAVY, S. Y SALES, G. (1991). Cooperative learning in computer based instruction. Educational Technology Research and Development, 39(2) 70-80.

STEEPLES, C. \& JONES, C., eds (2002). Networked Learning: Perspectives and Issues. London:Springer

TOURIÑAN, J.M. (2001). Tecnología digital y sistema educativo: el reto de la globalización. Revista de Educación. Monográfico Globalización y Educación. Ministerio de Educación, Cultura y Deporte. 
WATSON M.(2001). Pedagogy before Technology: Re-thinking the Relationship between ICT and Teaching. Education and Information Technologies, 6 (4): 251-266.

WEERT. T. J. Y PILOT, A. ( 2003). Task-Based Team Learning with ICT, Design and Development of New Learning. Education and Information Technologies.8 (2): 195-214.

\section{NOTAS}

[1] Esta aportación forma parte del plan de formación del profesorado en TICS dentro del proyecto I+D (Referencia : BSO2003-00340/PSCE) subvencionado por el Ministerio de Ciencia y Tecnología en convocatoria pública (BOE 31/10/2002) y aprobado en 2003( Notificación escrita 11 27/11/2003) para su ejecución "Utilización de las TICs en el desarrollo Curricular de la Educación Física: Diseño, Implementación y Evaluación de un modelo de formación para el Profesorado de Secundaria y Bachillerato" .Esta investigación la llevan a cabo los equipos de: "Investigación, Evaluación y Tecnología Educativa" ( CODIGO 154- H) y "Educación Física, Salud y Deporte", ambos pertenecientes al Plan Andaluz de Investigación.

[2] De especial interés resulta la consulta del monográfico de la revista Instructional Science, 2003, $\left.\mathrm{n}^{\mathrm{o}} .31\right)$.

(C) Ediciones Universidad de Salamanca 